\title{
Circulating immune complexes in cutaneous and systemic vasculitis: clinicopathological correlations
}

\author{
J. BARRATT, M. SINDREY, R. SUMMERLY, G. M. ABER, AND P. NAISH \\ From the Department of Postgraduate Medicine, University of Keele, North Staffordshire Medical Centre, \\ Stoke-on-Trent
}

SUMMARY A high incidence of raised levels of circulating immune complexes has been found in a group of patients with idiopathic vasculitis. Serial measurements in 8 patients with systemic vasculitis showed a good correlation between clinical status and circulating immune complex levels. Some correlation existed between the constituents of circulating and tissue-bound complexes in 6 patients so studied. However, there was no correlation between apparent organ involvement and hypocomplementaemia and the levels or size of circulating immune complexes. It is concluded that the measurement of circulating immune complexes may be clinically useful in vasculitis, but that no direct evidence is yet available that they are pathogenetically related to those which are tissue-bound.

It is generally accepted that vasculitis is produced by the deposition of circulating immune complexes (CIC) in blood vessel walls. This paper presents our findings relating to $\mathrm{CIC}$ in idiopathic cutaneous or systemic vasculitis. An attempt has been made to determine whether or not there is any correlation between the presence of CIC in the serum and the clinical state of the patients and the apparent organ involvement in the disease and also to relate the constituents of the CIC with the proteins present in the blood vessel walls.

\section{Material and methods}

Patients. A total of 36 patients were studied, and in 8 patients serial blood samples were obtained. Of the 36 patients 26 had apparently exclusive cutaneous vasculitis and 10 evidence of more generalised organ involvement. The diagnosis of vasculitis was made by clinical observation of a typical purpuric rash, confirmed by skin biopsy and, in the cases of systemic vasculitis, a renal biopsy. The division between cutaneous and systemic vasculitis was made on the basis of clinical examination and radiological and laboratory tests of lung, gut, or kidney involvement.

So far as possible only those patients with vasculitis of no known cause were included. Patients with systemic lupus erythematosus, rheumatoid

Accepted for publication 11 February 1981.

Correspondence to Dr P. Naish, Department of Nephrology, North Staffs Royal Infirmary, Princes Road, Stoke-on-Trent. arthritis, or similar 'connective tissue' diseases, and those with cutaneous vasculitis and renal-biopsyproved mesangial IgA deposition (Henoch-Schönlein purpura) were excluded. None of the patients included in the study had demonstrable hepatitis B antigenaemia.

Serum. Blood was allowed to clot at room temperature for 1-2 hours and centrifuged, and the separated serum was stored in aliquots at $-70^{\circ} \mathrm{C}$ until tested.

Immune complex assay. A radiolabelled monoclonal rheumatoid factor binding assay was used. ${ }^{1}$ Samples were assayed in duplicate and the result expressed as a percentage of total trichloracetic acid precipitable radioactivity. A calibration curve using heat aggregated IgG was performed with each batch of assays.

Serum complement component measurement. Serum C3 was measured by radial immunodiffusion with monospecific antisera (Hoechst). Results were expressed as a percentage of the value of pooled normal human serum.

Gel filtration of serum. $2 \mathrm{ml}$ of serum from 6 cases (4 cutaneous and 2 systemic vasculitis) was applied to a $65 \times 1.5 \mathrm{~cm}$ column of sepharose CL-6B (Pharmacia) previously calibrated with purified radiolabelled human IgM and IgG. $1 \cdot 7 \mathrm{ml}$ fractions were collected and assayed for rheumatoid factor binding activity. 1

Protein A-sepharose affinity chromatography. After rheumatoid factor binding (RFB) assay of fractions from the sepharose CL-6B column fractions 
were pooled as follows. Pool 1 consisted of those fractions from the void to fraction 28 , that is, 3 fractions before the peak of IgM elution. Pool 2 was taken from fractions $29-38$, that is, 3 fractions before

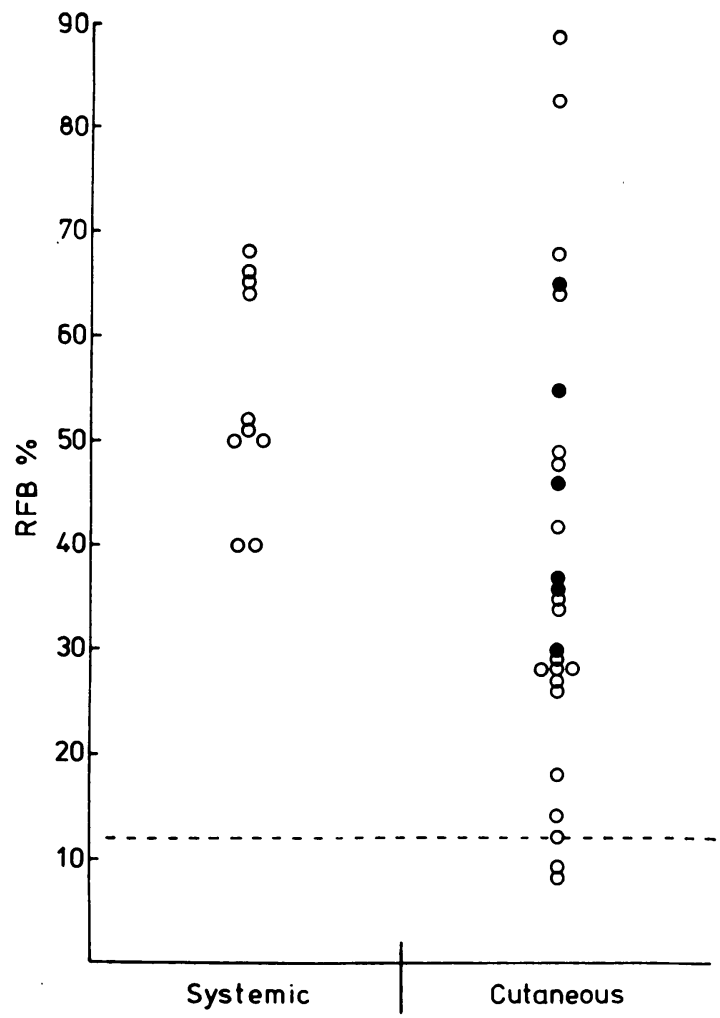

Fig. 1 Circulating immune complex levels (expressed as $\%$ rheumatoid factor binding) in patients studied. $0=$ Normocomplementaemic. $\bullet=$ Hypocomplementaemic. the peak of IgM elution to 4 fractions before the peak of IgG elution. Each pool was applied to a $4 \mathrm{ml}$ protein A-sepharose CL-4B column (Pharmacia) previously equilibrated with phosphate buffered saline $\mathrm{pH} 7 \cdot 2$ (PBS). The column was washed with PBS to remove all unbound protein. Protein bound to the column was eluted with $1.0 \mathrm{M}$ acetic acid. Eluted fractions $(1 \mathrm{ml})$ were pooled and neutralised with $1 \cdot 0 \mathrm{M} \mathrm{NaOH}$.

Analysis of material eluted from protein $A-$ sepharose column. Eluted material was tested for the presence of IgA, IgG, IgM, C3, C4, and $\mathrm{Clq}$ by Ouchterlony double diffusion in agarose and for C3 and $\mathrm{C} 4$ also by counterimmunoelectrophoresis (antisera from Hoechst).

Tissue biopsies. Skin biopsies were available in all 6 cases whose serum was studied as above. Renal biopsy was carried out in the 2 patients with systemic vasculitis. Portions for direct immunofluorescent staining were snap-frozen in liquid nitrogen, sectioned at $4 \mu \mathrm{m}$, and stained by routine methods using fluorescein conjugated antisera to $\operatorname{IgG}, \operatorname{IgA}$, IgM, C1q, C4, C3, and fibrin (Hoechst).

\section{Results}

Circulating immune complexes and disease manifestations (Fig. 1). Sera from 31 of 36 patients with vasculitis showed unequivocally raised RFB. The RFB value bore no relationship to presence or absence of hypocomplementaemia or clinical and laboratory evidence of organ involvement. In 2 patients with apparently exclusive cutaneous vasculitis and hypocomplementaemia a raised RFB was observed in both the presence and the absence of a rash.

Serial studies (Fig. 2). During the treatment with steroids resulting in a favourable response in 8

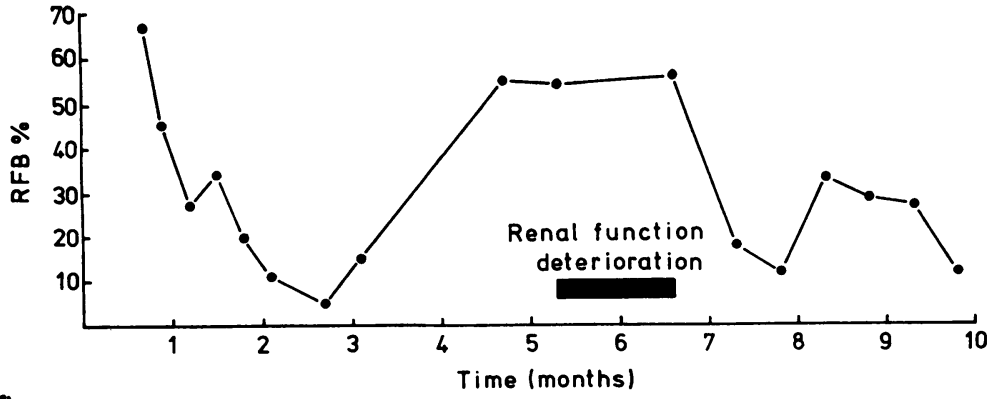

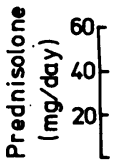

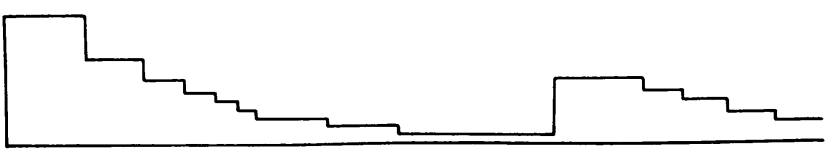

Fig. 2 Serial circulating immune complex measurements in a patient with systemic vasculitis.

Recurrence of disease on reduction of prednisolone was controlled by increased dosage. 


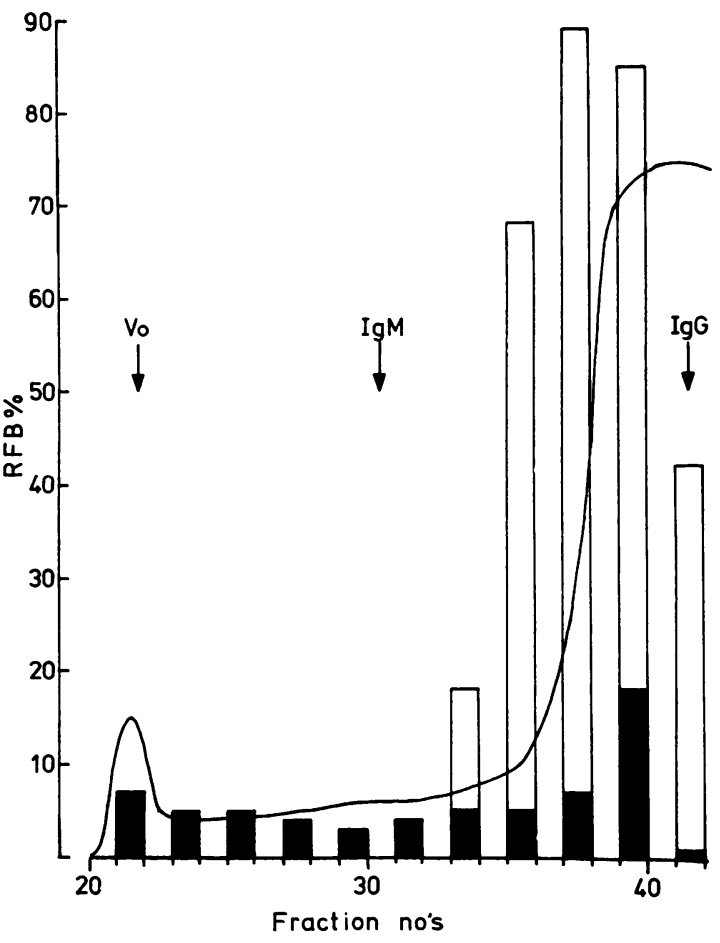

Fig. 3 Rheumatoid factor binding of fractions from sepharose $C L-6 B$ column (after substraction of RFB value obtained from normal human serum). Exclusion peak (Vo) and elution positions of IgM and IgG are shown. $\square=$ Case 4. $\Pi=$ Case 1 .

patients with systemic vasculitis RFB returned to normal in parallel with clinical response in 7 cases. This association was not observed in the eighth patient, who presented as a classical case of polyarteritis nodosa with evidence of pulmonary, renal, cutaneous, and joint involvement. Response to steroid therapy in this patient was rapid, and all evidence of active disease had disappeared within 2 weeks. Disease recurred in 3 patients during the study. In each case this was accompanied by a rise in RFB and a subsequent fall during response to raised steroid dosage.

Sepharose CL-6B fractionation of serum (Fig. 3). Two representative examples of RFB assay results of gel filtration fractions are shown. Two cases of cutaneous and both cases of systemic involvement showed material of molecular weight $<c .1 \cdot 10^{6}$ as well as between $1 \cdot 10^{6}$ and $3 \cdot 10^{5}$ daltons. The other 2 cases of cutaneous vasculitis (cases 1 and 2) showed only $c$. $5-3 \cdot 10^{5}$ dalton material (these findings are summarised in Table 1).

Protein-A sepharose column analysis (Table 1). Analysis of pools 1 and 2 from normal human serum showed IgG only in pool 2. IgA was not detected in pool 1 in any of the vasculitis patients but was present in all in pool 2, as was IgG. IgG and IgM were found in pool 1 in 1 case of cutaneous and 1 of systemic vasculitis, and IgM was present in pool 2 of both these patients and 1 other with cutaneous vasculitis. C3 and C4 were not detected in either pool from any patient.

\section{TISSUE IMMUNOFLUORESCENCE (Table 2)}

Skin biopsies. IgM, C1q, and $\mathrm{C} 3$ and were seen in blood vessel walls in all cases. IgG was present in this location in 2 patients with cutaneous and 1 with systemic vasculitis, and $\operatorname{IgA}$ was present in 2 patients with cutaneous and both patients with systemic involvement.

Table 2 Immunoglobulin deposition in blood vessel walls

\begin{tabular}{ccccccc}
\hline & \multicolumn{5}{c}{ Skin biopsy } & \multicolumn{5}{c}{ Kidney biopsy } \\
\cline { 2 - 7 } Patient & IgG & IgA & IgM & IgG & IgA & IgM \\
\hline $\mathbf{1}$ & - & + & + & & & \\
$\mathbf{2}$ & - & - & + & & ND & \\
$\mathbf{3}$ & + & - & + & & & \\
$\mathbf{4}$ & + & + & + & + & + & + \\
$\mathbf{5}$ & - & + & + & + & + & + \\
$\mathbf{6}$ & + & + & + & + & + & \\
\hline
\end{tabular}

ND = kidney biopsy not done.

Table 1 Immunoglobulin content of protein A-sepharose eluates together with details of organ involvement, serum $C 3$ (expressed as \% pooled normal human serum), and presence of raised RFB in pooled fractions of sepharose CL-6B column

\begin{tabular}{|c|c|c|c|c|c|c|c|c|c|c|}
\hline \multirow[b]{2}{*}{ Patient } & \multirow{2}{*}{$\begin{array}{l}\text { Organ } \\
\text { involvement }\end{array}$} & \multirow{2}{*}{$\begin{array}{l}\text { Serum } \\
\text { C3 }\end{array}$} & \multicolumn{4}{|c|}{ Pool 1} & \multicolumn{4}{|c|}{ Pool 2} \\
\hline & & & $R F B$ & $\operatorname{Ig} G$ & $\operatorname{Ig} A$ & $\operatorname{Ig} M$ & $R F B$ & $I g G$ & $\operatorname{Ig} A$ & $I g M$ \\
\hline 1 & Skin & 100 & - & - & - & - & $\uparrow$ & + & + & - \\
\hline 2 & Skin & 100 & - & + & - & + & $\uparrow$ & + & + & + \\
\hline 3 & Skin & 42 & $\uparrow$ & - & - & - & $\uparrow$ & + & + & + \\
\hline 4 & Skin & 133 & $\uparrow$ & - & - & - & $\uparrow$ & + & + & - \\
\hline 5 & Systemic & 85 & $\uparrow$ & - & - & - & $\uparrow$ & + & + & - \\
\hline 6 & Systemic & 121 & $\uparrow$ & + & - & + & $\uparrow$ & + & + & + \\
\hline \multicolumn{2}{|c|}{ Normal human serum } & $70-120$ & - & - & - & - & - & + & - & - \\
\hline
\end{tabular}


Kidney biopsies. These were available from both patients with systemic involvement and showed IgM, $\mathrm{Clq}$, and $\mathrm{C} 3$ in blood vessel walls and IgG, IgM, and $\mathrm{C} 3$ deposited in a granular pattern on the glomerular basement membrane.

\section{Discussion}

This study confirms the findings of other groups that circulating immune complexes are detectable in a high proportion of patients with vasculitis. ${ }^{2-4}$ The incidence and amount of circulating complexes did not, however, appear to be related to clinical and laboratory evidence of organ involvement or to the extent of complement activation. On the other hand serial measurement of CIC in 8 patients with systemic vasculitis showed a fall to normal of CIC levels in serum in association with a favourable response to prednisolone treatment. In 3 of these patients a relapse of the condition was associated with a rise in CIC and a subsequent fall with increased prednisolone dosage.

The serial measurements of CIC during treatment of systemic vasculitis reported here expands the information already available from this laboratory. ${ }^{5}$ There are no other reports of such studies, and the findings may be taken as further evidence for a pathogenetic role of CIC in vasculitis. Nevertheless the lack of correlation between CIC levels and extent of organ involvement raises the possibility that such a conclusion may be too simplistic. One of the patients with systemic vasculitis who was treated with prednisolone showed raised CIC for 2 months despite prompt and maintained response to treatment, and in addition 2 of the cases of cutaneous vasculitis were studied in the presence and in the absence of a rash and there was no difference in the levels of CIC. Thus under some circumstances CIC may be present, but do not appear to cause vascular inflammation.

A number of factors have been shown to determine whether or not CIC deposit in tissues in experimental models of immune complex disease. These include size of $\mathrm{CIC}^{6}$ and complement-mediated changes. ${ }^{7}$ Molecular size analysis of CIC from serum of 6 of our patients (4 with cutaneous and 2 with systemic vasculitis) did not show any discernible differences according to organ involvement. Previous studies have found mostly $>19 \mathrm{~S}$ CIC in vasculitis. ${ }^{2-4}$ Four of our patients showed similar findings. It is possible that the CIC in the other 2 patients dissociated to some extent on gel filtration, resulting in the larger amount of RFB material eluting just before IgG. There is no evidence that our monoclonal rheumatoid factor reacts preferentially with com- plexes of a particular molecular size, ${ }^{5}$ but it is IgG specific. It is thus also possible that predominantly IgM-containing CIC were present in the 2 patients in whom no $>1 \cdot 10^{6}$ dalton RFB material was found. Some evidence for the existence of such CIC is available from the study of Burden et al. ${ }^{8}$

Kaufman et al. ${ }^{4}$ showed that a good correlation existed between the constituents of circulating and tissue immune complexes in some forms of vasculitis. We were able to analyse the constituents in only a small number of patients with high RFB serum. Gel filtration of serum results in quite marked dilution, and a high RFB in serum is required to detect such material in column fractions, Kaufman et al. ${ }^{4}$ used a different CIC assay system and method of analysing the constituents of $\mathrm{CIC}$, and a direct comparison with our findings is thus difficult. The use of protein $A$ as a ligand for isolation of CIC suffers from the drawback that IgG subclass 3 containing material is not bound, ${ }^{9}$ and may in part explain the failure to demonstrate complement components in any of the protein A eluates. In addition CIC which did not contain IgG would not have been detected by the RFB assay or bound on the protein A-sepharose column. Such CIC may have been present in patients 1,4 , and 5 in whose protein A-sepharose eluates IgM was not found. The finding of IgG in renal or cutaneous blood vessel walls in vasculitis is rare in our laboratory, but IgG is frequently found in glomeruli of patients with systemic vasculitis with renal involvement. In addition $\mathrm{IgM}, \mathrm{C} 3$, and $\mathrm{Clq}$ are demonstrable in intrarenal blood vessel walls not only of patients with vasculitis but also of those with accelerated hypertension and with hypertension associated with pregnancy or oral contraceptives. Thus the presence of these proteins may be a nonspecific manifestation of damage to blood vessel walls caused by a number of stimuli. Increased vascular permeability exists in association with vasculitis, and thus the finding of $\operatorname{IgM}$ and $\operatorname{IgA}$ in both blood vessel walls and in CIC in this study and in that of Kaufman et al. ${ }^{4}$ does not necessarily establish evidence of a direct pathogenetic relationship.

In conclusion, measurement of CIC in vasculitis by the IgG-specific monoclonal RFB assay, while it frequently showed abnormal results and correlated well with disease activity in systemic vasculitis, did not discriminate between patients with differing extent of organ involvement. The constituents of CIC bore some relation to proteins in blood vessel walls, but no direct evidence exists that they were pathogenetically related to vascular inflammation. Thus the measurement of CIC in patients with vasculitis may be clinically useful, particularly if 
considered together with the results of other tests of disease activity, but it is not yet possible to be certain of their direct pathogenetic role.

J. Barratt and M. Sindrey were supported by the Medical Research Council.

\section{References}

1 Barratt J, Naish. A simple radiolabelled rheumatoid factor binding assay for the measurement of circulating immune complexes. J Immunol Methods 1979; 25: 137-46.

2 Mackel S E, Tappeiner G, Brumfield H, Jordon R E. Circulating immune complexes in cutaneous vasculitis. $J$ Clin Invest 1979; 64: 1652-60.

3 Kammer G M, Soter N A, Schur P H. Circulating immune complexes in patients with necrotizing vasculitis. Clin Iminunol Immunopathol 1980; 15: 658-72.

4 Kaufman R H, Herrmmann W A, Meijer C J L M, Daha M R, Van Es L A. Circulating and tissue bound immune complexes in allergic vasculitis: relationship between immunoglobulin class and clinical features. Clin Exp Immunol 1980; 41 : 459-70.

5 Naish P, Barratt J, Sindrey M. Assay of soluble immune complexes using radiolabelled rheumatoid factor. Methods Enzymol in press.

6 Cochrane C G, Hawkins D. Studies on circulating immune complexes. III. Factors governing the ability of circulating complexes to localize in blood vessels. $J$ Exp Med 1968; 127: 137-54.

7 Henson P M, Cochrane C G. Immune complex disease in rabbits. The role of complement and of a leukocyte dependent release of vasoactive amine from platelets. $J$ Exp Med 1971; 133: 554-71.

8 Burden R P, Cotton C E, Wallington T B, Reeves W G. Immune deposits in extraglomerular vessels: their correlation with circulating immune complexes. $J$ Exp Immunol 1980; 42: 483-9.

9 Kronvall G, Williams R C. Differences in anti-protein A activity among IgG subgroups. J Immunol 1969; 103: 828-33. 